\title{
An Accurate Modulation Recognition Method of QPSK Signal
}

\author{
Yongxin Feng, Zhenyu Teng, Fanwei Meng, and Bo Qian \\ School of Information Science and Engineering, Shenyang Ligong University, Shenyang 110159, China \\ Correspondence should be addressed to Yongxin Feng; fengyongxin@263.net
}

Received 5 November 2014; Revised 21 April 2015; Accepted 19 May 2015

Academic Editor: Chih-Cheng Hung

Copyright (C) 2015 Yongxin Feng et al. This is an open access article distributed under the Creative Commons Attribution License, which permits unrestricted use, distribution, and reproduction in any medium, provided the original work is properly cited.

\begin{abstract}
Quadrature phase shift keying (QPSK) modulation has been widely applied in communication systems. With the increasing development of QPSK modulation recognition, it is meaningful for ensuring validity and accuracy of recognition method. Therefore, the method of signal recognition has been presented based on the features of QPSK modulated signal, in which the feature parameters of QPSK signal are extracted. Besides, signal classification is fulfilled through thresholds, and modulation recognition is completed with quartic spectrum. The simulation results show that, the method can recognize QPSK modulation effectively in less sample space condition and can be more accurate.
\end{abstract}

\section{Introduction}

Modulation recognition is the technology which can determine the modulation by analyzing the received signal in the premise of unknown signal modulation (or only a little known priori information). Modulation recognition technology has been widely used in the communication field [15], such as software radio platform and electronic warfare technology.

The QPSK modulated signal has the features of low error rate, strong antijamming ability, and low complexity [6], so it is widely used in the intersatellite communications, such as GPS navigation systems, BeiDou navigation systems [7], and common data links (CDL) [8]. Recognition methods for QPSK modulated signal can be categorized into two kinds: the time-domain feature parameter methods and the highorder cumulants methods [9-11]. However, the performances of both methods are constrained by the number of sample space, and neither of them considers how to distinguish the QPSK modulated signals from the MPSK modulated ones.

In this paper, we focus on the need for recognizing the QPSK modulation and present a method based on extracting the feature parameter of the signal, which can distinguish the QPSK modulated signals from the MPSK modulated ones. Details on analyzing the quartic spectrum of QPSK signal and validating the feasibility of the method are discussed in the later sections.

\section{QPSK Signal and Feature Parameters}

2.1. QPSK Signal Model. A QPSK signal can be expressed as

$$
s(t)=A \cos \left(\omega_{0} t+\theta_{k}\right), \quad k=1,2,3,4,
$$

where $A$ is the signal amplitude; $\omega_{0}$ is the carrier frequency; $\theta_{k}, k=1,2,3,4$, are set as $0, \pi / 2, \pi$, and $3 \pi / 2$, respectively, and the selection of $\theta_{k}$ is determined by the value of the baseband code.

Table 1 shows the relationship between the base-band code and $\theta_{k}$. Each QPSK base-band code contains 2-bit information, which is represented by the symbol $a b$. There are four statuses in the symbol $a b$, namely, 00, 01, 11, and 10 . Each status is represented by one of $\theta_{k}, k=1,2,3,4$. The various relationships between the phase values are designed according to the Gray code [12].

2.2. Feature Parameters. Since the phase value of QPSK is controlled by the base-band code, the change of the amplitude information can be indicated by the change of phase values. The nonamplitude modulated signal, such as 2FSK, MFSK, and MSK signals, can be distinguished from QPSK signal through this feature. The QPSK signal contains the information of the absolute phase, so the BPSK and ASK modulation without the information of the absolute phase can be distinguished from QPSK signal through this feature. The instantaneous envelope's mean of the QPSK signal is 
TABLE 1: The relationship between the base-band code and $\theta_{k}$.

\begin{tabular}{lcccc}
\hline$a b$ & 00 & 01 & 11 & 10 \\
\hline Phase values $\theta_{k}$ & 0 & $\pi / 2$ & $\pi$ & $3 \pi / 2$ \\
\hline
\end{tabular}

close to 1, while the instantaneous envelope's mean of the QAM signal changes in a relatively large range, so QAM can be distinguished from QPSK signal through this feature. Above all, three feature parameters are needed to recognize the QPSK modulations $[13,14]$.

(1) The maximum value of the spectral power density of the normalized-centered instantaneous amplitude $R_{\max }$ of the signal is given by

$$
R_{\max }=\frac{\operatorname{MAX}\left(\left|\operatorname{FFT}\left(a_{\mathrm{cn}}(i)\right)\right|^{2}\right)}{N_{s}},
$$

where $a_{\mathrm{cn}}(i)$ is the normalized-centered instantaneous amplitude and $N_{s}$ is the number of samples per block.

(2) The standard deviation of the absolute value of the centered nonlinear component of the instantaneous phase, evaluated over the nonweak intervals $\delta_{a p}$ of the signal, is given by

$$
\delta_{a p}=\sqrt{\frac{1}{c}\left[\sum_{a_{n}(i)>a_{t}} \varphi_{\mathrm{NL}}^{2}(i)\right]-\left[\frac{1}{c} \sum_{a_{n}(i)>a_{t}}\left|\varphi_{\mathrm{NL}}(i)\right|\right]^{2}}
$$

where $a_{t}=1$ is a threshold which determinates the nonweak signal; $c$ is the number of samples in $\varphi_{\mathrm{NL}}$ for which $a_{n}(i)>a_{t}$; and $\varphi_{\mathrm{NL}}$ is the centered-nonlinear components of instantaneous phase.

(3) The average value of the instantaneous amplitude $E_{a}$ is given by

$$
E_{a}=\frac{1}{N_{s}} \sum_{n=1}^{N_{s}} a(n)
$$

where $a(n)$ is the instantaneous amplitude and $N_{s}$ is the number of samples per block.

\section{Quartic Spectrum and Modulation Recognition Process}

3.1. Quartic Spectrum Analysis of QPSK Signal. In this section, quartic spectrum of QPSK signal is analyzed to determine the modulation. Since four-phase values exist in the QPSK modulation, discrete spectral lines will appear near the quadruple frequency [15] after the signal is processed by quartic spectrum and FFT processing, which is significantly different from other MPSK $(M \geq 8)$ signals. The QPSK signal in (1) is squared, which can be expressed as

$$
S^{2}(t)=\frac{A^{2}}{2}+\frac{A^{2}}{2} \cos \left(2 \omega_{0} t+2 \theta_{k}\right), \quad k=1,2,3,4 .
$$

In order to remove the DC component, a high-pass filter is used after square processing. After another square
TABLE 2: The relationship between the base-band code and phase of 8PSK.

\begin{tabular}{lcccccccc}
\hline \multirow{3}{*}{ Code } & 0 & 0 & 0 & 0 & 1 & 1 & 1 & 1 \\
& 0 & 0 & 1 & 1 & 1 & 1 & 0 & 0 \\
& 0 & 1 & 1 & 0 & 0 & 1 & 1 & 0 \\
\hline Phase values $\theta_{k}$ & 0 & $\pi / 4$ & $\pi / 2$ & $3 \pi / 4$ & $\pi$ & $5 \pi / 4$ & $3 \pi / 2$ & $7 \pi / 4$ \\
\hline
\end{tabular}

operation, the quartic spectrum of QPSK modulated signal is got, which can be expressed as follows:

$$
S^{4}(t)=\frac{A^{4}}{8}+\frac{A^{4}}{8} \cos \left(4 \omega_{0} t+4 \theta_{k}\right), \quad k=1,2,3,4,
$$

where the phase values $4 \theta_{k}$ will be $0,2 \pi, 4 \pi$, and $6 \pi$, respectively.

Then, (6) can be written simply as

$$
S^{4}(t)=\frac{A^{4}}{8}+\frac{A^{4}}{8} \cos \left(4 \omega_{0} t\right), \quad k=1,2,3,4 .
$$

From (7), we can conclude that the discrete spectral lines of QPSK signals will appear at quadruple frequency $4 \omega_{0}$ after the quartic spectrum and FFT processing. For MPSK $(M \geq 8)$ modulation signal, $M$ phase values are used. For example, the phase values of 8PSK signal are shown in Table 2.

For $8 \mathrm{PSK}$, after the quartic spectrum and FFT processing, the value of $4 \theta_{k}$ modulo $2 \pi$ is 0 or $\pi$, so the discrete spectral lines at the quadruple frequency will not appear. According to this, the QPSK modulation can be distinguished from the 8PSK (MPSK, $M \geq 8$ ) one.

3.2. Modulation Recognition Process. The modulation recognition process of QPSK signal is shown in Figure 1.

Step 1. Three feature parameters of the signal being recognized are extracted successively, including the maximum value of the spectral power density of the normalizedcentered instantaneous amplitude $R_{\max }$, the standard deviation of the absolute value of the centered nonlinear component of the instantaneous phase, evaluated over the nonweak intervals $\delta_{a p}$, and the average value of the instantaneous amplitude $E_{a}$.

Step 2. The feature parameters are compared with the threshold value set before. If the conditions $R_{\max }>t\left(R_{\max }\right), \delta_{a p}>$ $t\left(\delta_{a p}\right)$, and $E_{a}>t\left(E_{a}\right)$ cannot be met, it can be concluded that the signal being recognized is not a QPSK modulated one and the recognition process will end.

Step 3. The quartic spectrum of the received signal is calculated. If the discrete spectral lines at the quadruple frequency do exist, the modulation of the received signal will be determined as QPSK.

\section{Simulation}

Simulation is performed to verify the correctness and effectiveness of the modulation recognition method. The simulation parameters are set as follows: the carrier wave frequency 


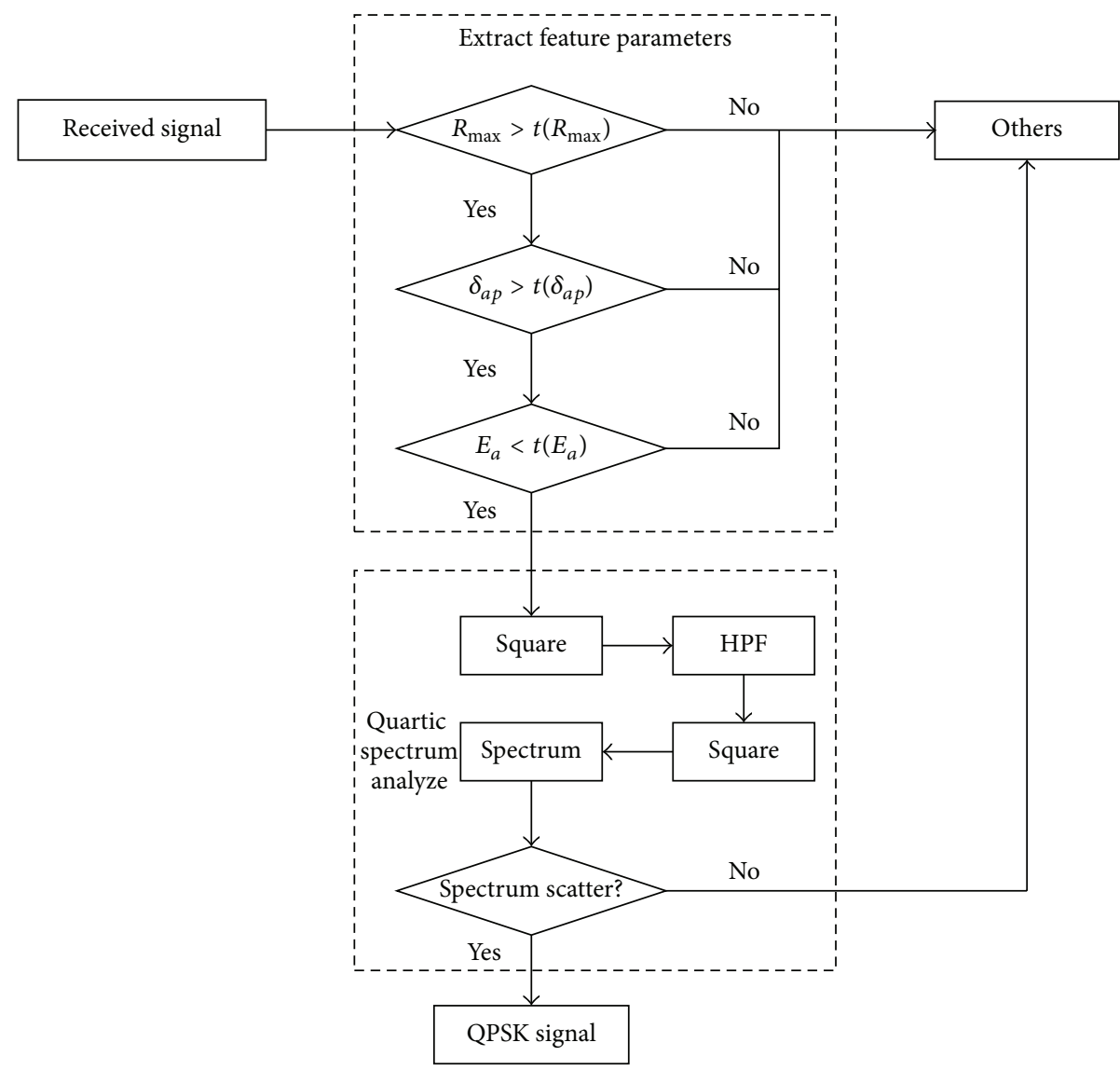

FIGURE 1: Modulation recognition process of QPSK signal.

is $102.3 \mathrm{MHz}$; the code frequency is $10.23 \mathrm{MHz}$; the sampling frequency is $920.7 \mathrm{MHz}$; and the number of sampling points is 18000 . Because the reasonable threshold values are closely related to the simulation parameters above, a large number of simulations and tests are performed to get the prior knowledge and the threshold values are set as $t\left(R_{\max }\right)=3.0$, $t\left(\delta_{a p}\right)=0.6$, and $t\left(E_{a}\right)=1.2$.

According to the first step of the recognition process, three feature parameters are calculated. Firstly, the maximum value of the spectral power density of the normalizedcentered instantaneous amplitude $R_{\max }$ is calculated. Figure 2 shows a plot of $R_{\max }$ versus SNR. It can be clearly found that the calculated feature parameter $R_{\max }$ of QPSK signal can meet the condition of $R_{\max }>t\left(R_{\max }\right)$. Meanwhile, $R_{\max }$ of MSK and QPSK signals is calculated under the same SNR condition, and the results are shown in Figure 3. It can be clearly found that $R_{\max }$ of QPSK signal can meet the condition of $R_{\max }>t\left(R_{\max }\right)$, while the $R_{\max }$ of MSK cannot, which prove the rationality of the threshold $t\left(R_{\max }\right)$. Therefore, the QPSK modulation can be distinguished from the MSK one by using the parameter $R_{\max }$.

Secondly, the standard deviation of the absolute value of the centered nonlinear component of the instantaneous phase, evaluated over the nonweak intervals $\delta_{a p}$, is calculated. Figure 4 shows a plot of $\delta_{a p}$ versus SNR. It can be clearly found that he calculated feature parameter $\delta_{a p}$ can meet the

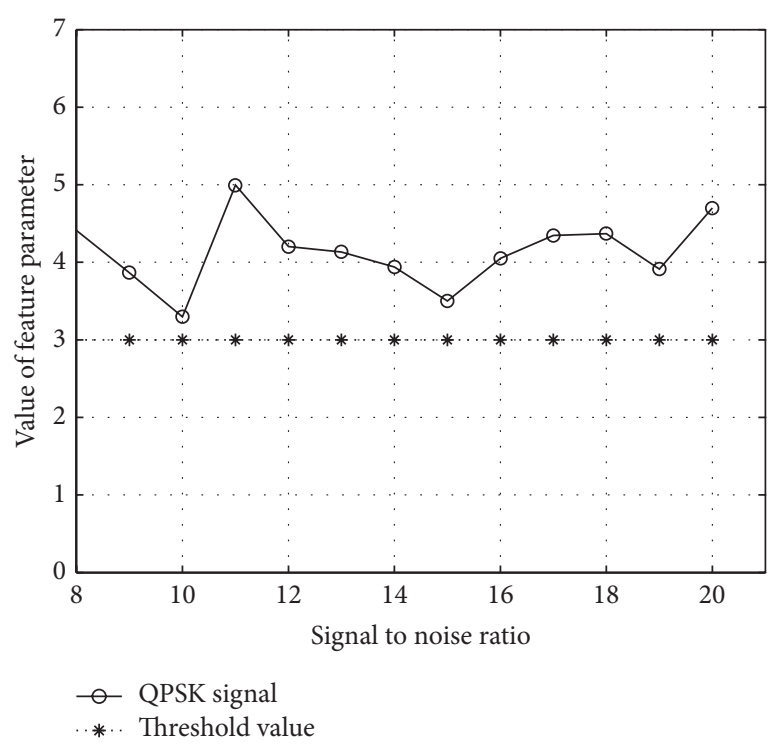

FIGURE 2: The $R_{\max }$ of QPSK signal.

conditions of $\delta_{a p}>t\left(\delta_{a p}\right)$. Meanwhile, the same feature parameter $\delta_{a p}$ of BPSK and QPSK signals is calculated under the same SNR condition, and the results are shown in 


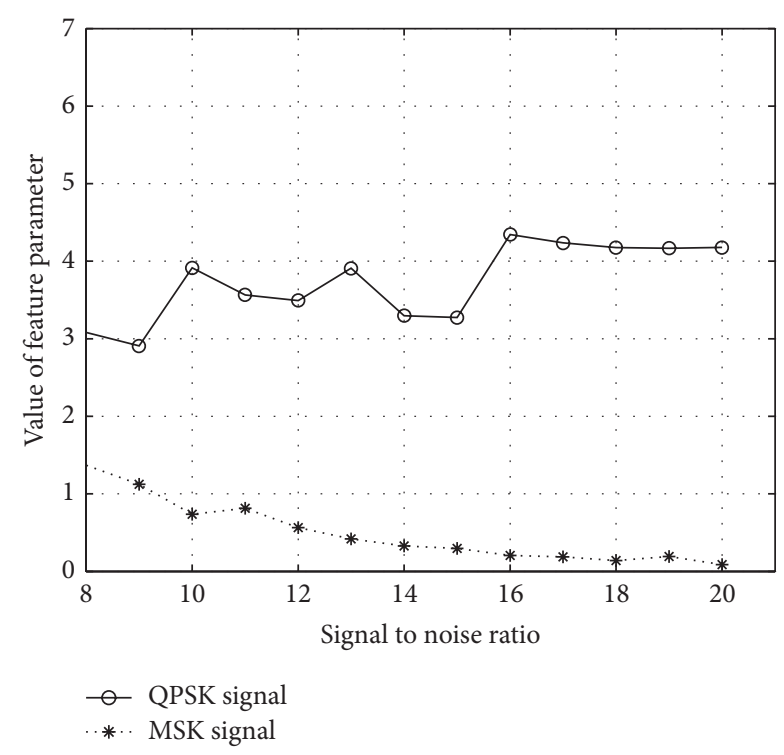

FIGURE 3: The $R_{\max }$ of QPSK and MSK signals.

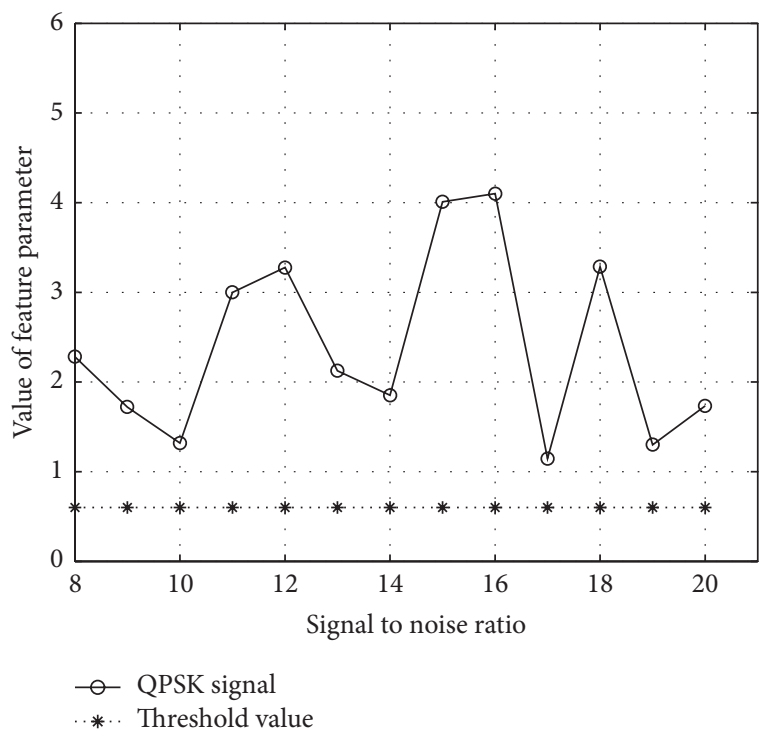

FIgURE 4: The $\delta_{a p}$ of QPSK signal.

Figure 5. It can be found that the $\delta_{a p}$ of QPSK signal can meet the condition of $\delta_{a p}>t\left(\delta_{a p}\right)$, while the $\delta_{a p}$ of BPSK cannot, which prove the rationality of the threshold $t\left(\delta_{a p}\right)$. Therefore, the QPSK modulation can be distinguished from the BPSK one by using the parameter $\delta_{a p}$.

Thirdly, the average value of the instantaneous amplitude $E_{a}$ is calculated. Figure 6 shows a plot of $t\left(E_{a}\right)$ versus SNR. It can be clearly found that he calculated feature parameter $E_{a}$ can meet the conditions of $E_{a}>t\left(E_{a}\right)$. Meanwhile, the same feature parameter $E_{a}$ of 16QAM and QPSK signals is calculated under the same SNR condition, and the results are shown in Figure 7. It can be found that the $E_{a}$ of QPSK signal can meet the condition of $E_{a}>t\left(E_{a}\right)$, while the $E_{a}$ of 16QAM cannot, which prove the rationality of the threshold $t\left(E_{a}\right)$.

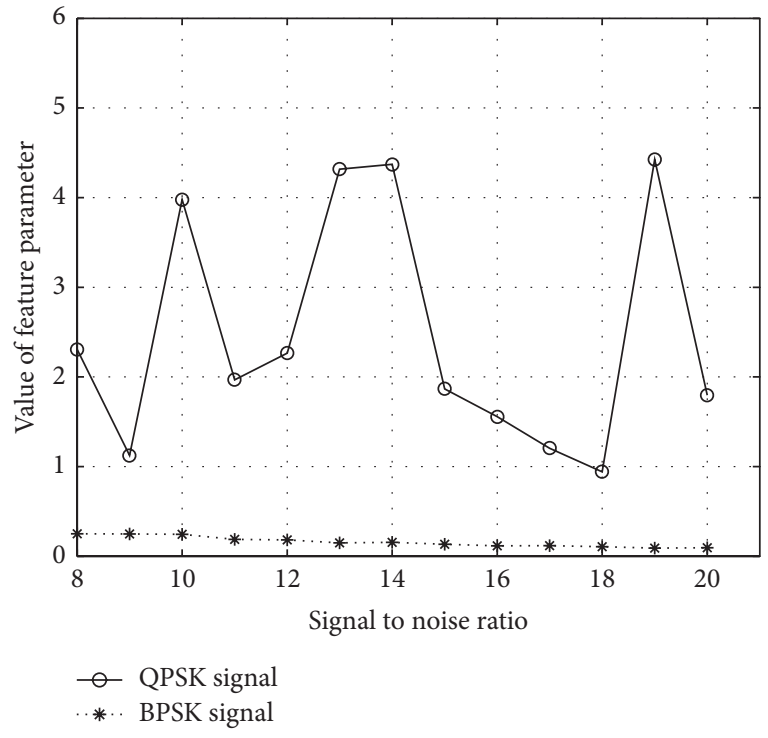

FIGURE 5: The $\delta_{a p}$ of QPSK and BPSK signals.

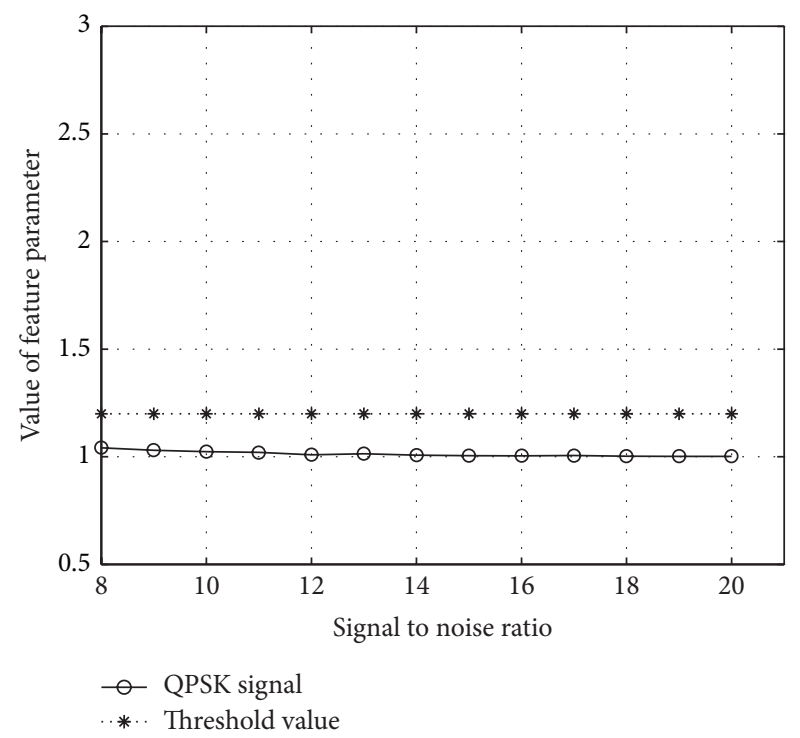

FIgURE 6: The $E_{a}$ of QPSK signal.

Therefore, the QPSK modulation can be distinguished from the 16QAM one by using the parameter $E_{a}$.

According to the second step of the recognition process, the modulation of signals can be determined. Due to the high similarity between QPSK and MPSK signals $(M \geq 8)$, the quartic spectrum of the signals is analyzed to distinguish them. Take the 8PSK modulation as an example of the MPSK signals. Figures 8 and 9 show the quartic spectrum of QPSK and 8 PSK signals, respectively, on condition that the SNR is $8 \mathrm{~dB}$.

Figure 8 indicates that a discrete spectrum line does exist at the quadruple frequency of the QPSK signal, while Figure 9 indicates that no discrete spectrum lines exist at the quadruple frequency of the 8PSK signal. By calculating 

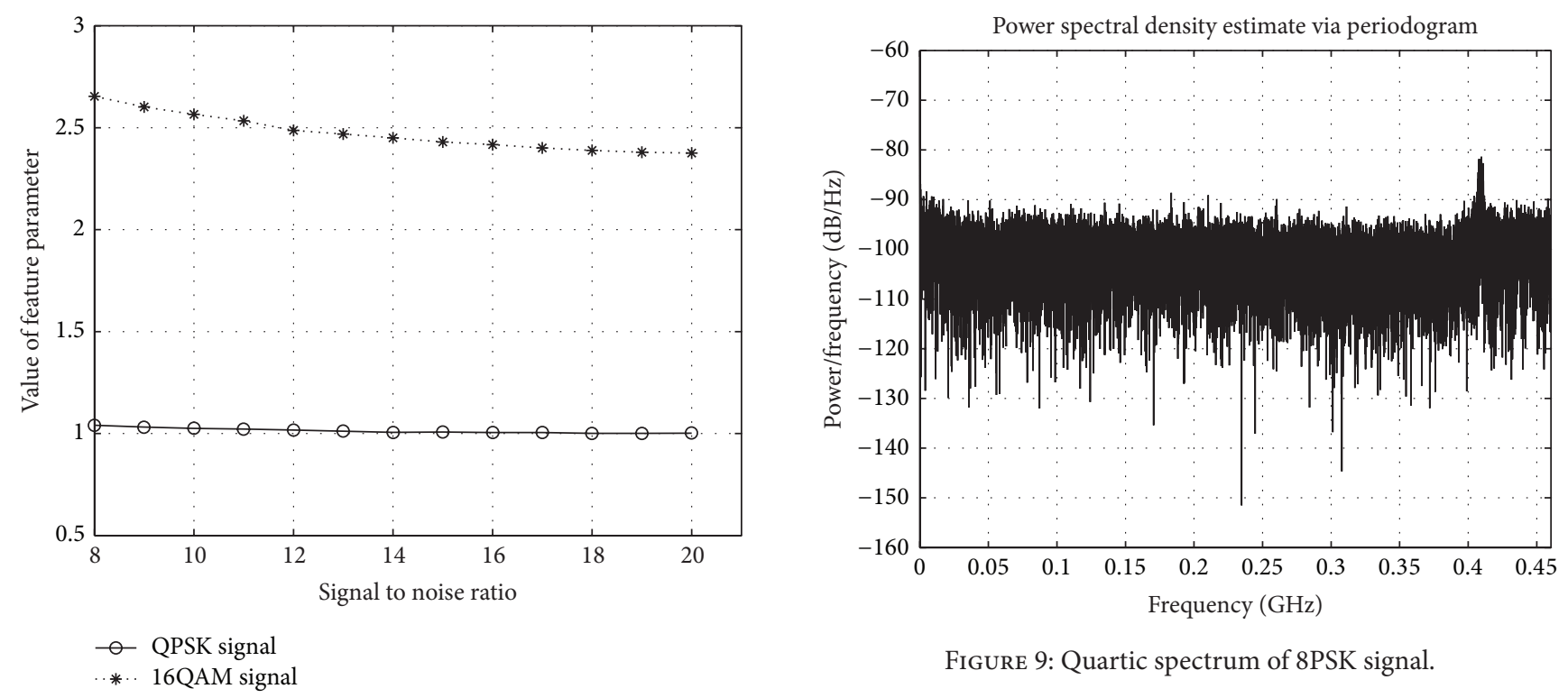

FIGURE 9: Quartic spectrum of 8PSK signal.

FIGURE 7: The $E_{a}$ of QPSK and 16QAM signals.
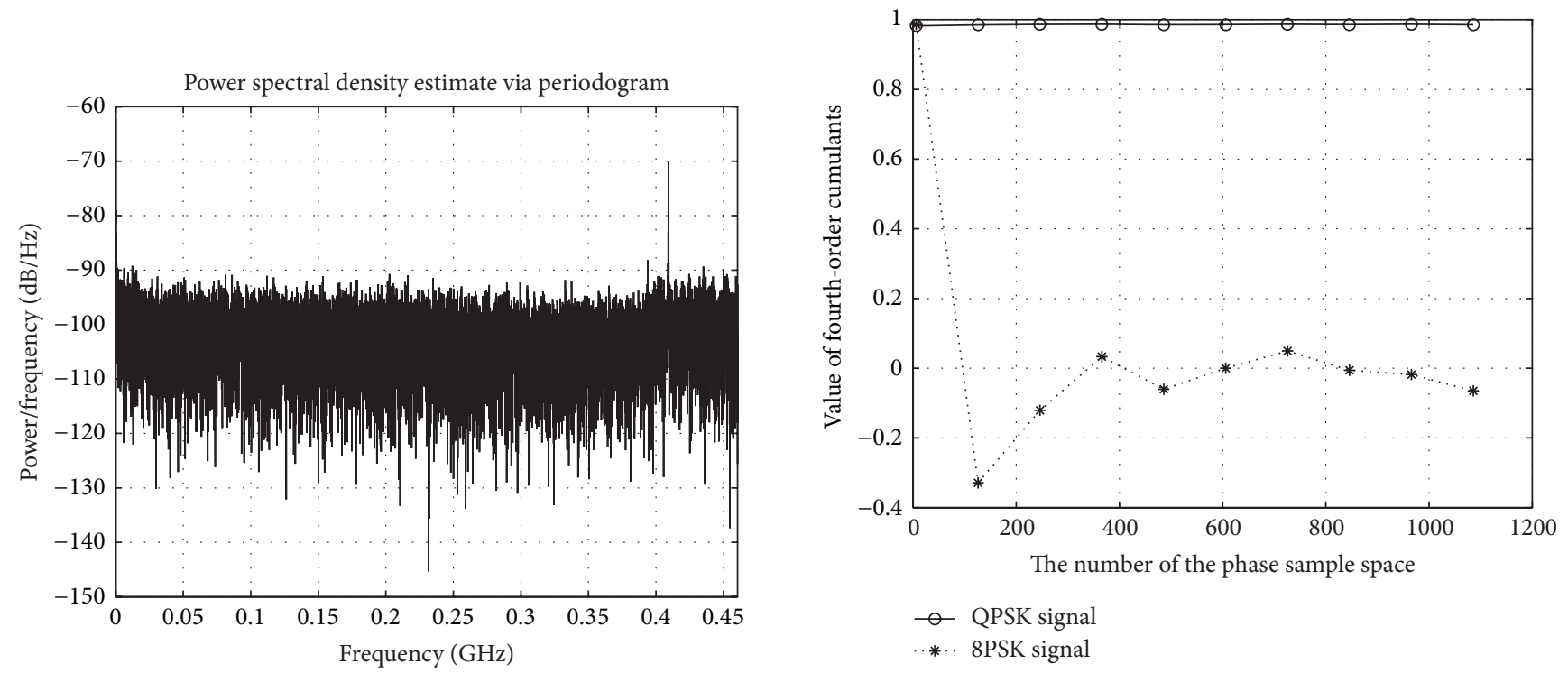

FIGURE 8: Quartic spectrum of QPSK signal.

the ratio of the maximum peak value to the mean value in the quartic spectrum and comparing it with the threshold values, it can be determined whether the signal is QPSK modulated or not.

Another simulation is performed to test the performance of the proposed method which is influenced by the number of phase sample space. As a comparison, a simulation of the method based on fourth-order cumulants under the same conditions is also performed. The simulation results are shown in Figures 10 and 11, in which the SNR is $10 \mathrm{~dB}$.

Figure 10 indicates that, for the method based on fourthorder cumulants, when the number of the phase sample space is more than 200, QPSK signal can be recognized effectively, while when the number of the phase sample space is less than 200, it is difficult to distinguish between QPSK modulation

and 8PSK modulation. This is because the method based on fourth-order cumulants can work well only when the sample values of phase space appear with a similar probability, which cannot be guaranteed when the number of phase sample is insufficient.

Figure 11 indicates that, for the proposed method, the QPSK signal can be distinguished easily form the 8PSK signal as long as the number of phase sample space is greater than 30 . So the proposed method can still work well when the number of the phase sample space is less than 200 (as long as it is larger than 30), which cannot be done by the method based on fourth-order cumulants. This is because, in the proposed method, the quartic spectrum peak proportion is calculated by energy integration, which does not need a large number of phase samples. 


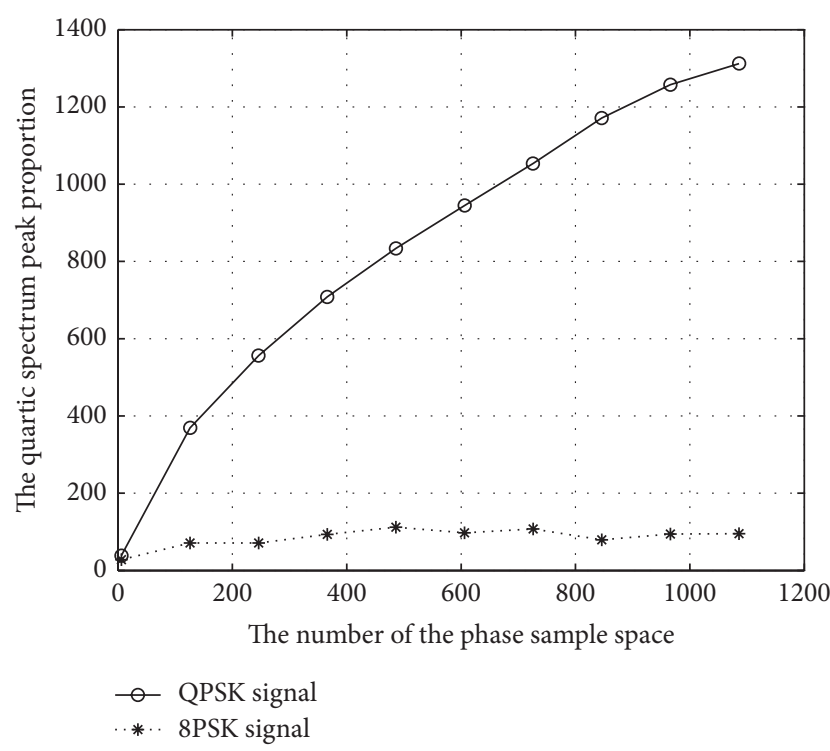

FIGURE 11: The quartic spectrum peak proportion of QPSK and 8PSK signals.

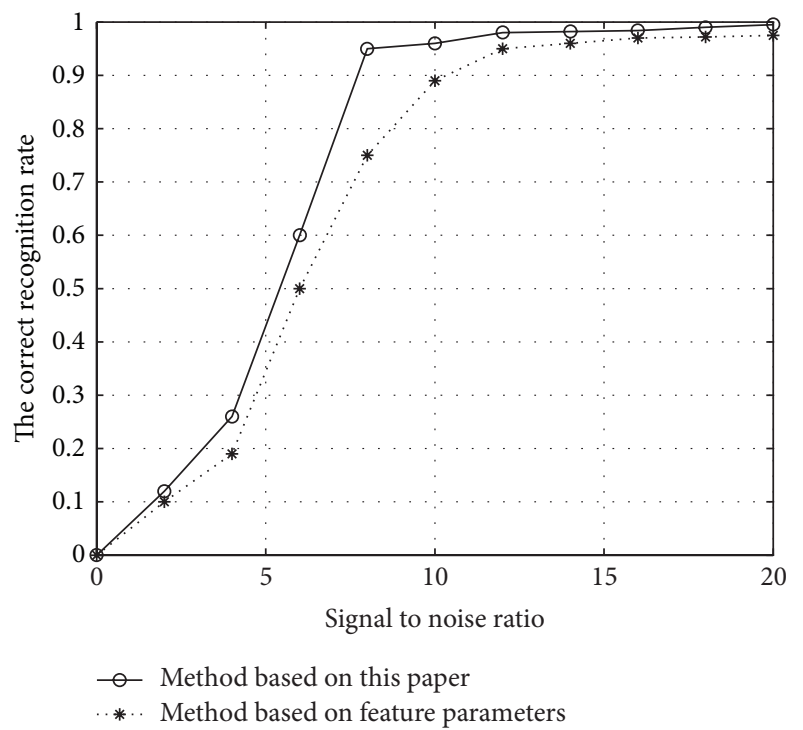

FIGURE 12: The correct recognition rate of methods.

Simulations are also performed to test the correct recognition rate of the proposed method and the traditional method based on feature parameters. The result is shown in Figure 12. It can be found that the correct recognition rate of the proposed method is greater than that of the traditional method based on feature parameters, especially for the SNR range between $8 \mathrm{~dB}$ and $10 \mathrm{~dB}$.

\section{Conclusion}

We presented a modulation recognition method of QPSK signal which is based on the combination of feature parameters and the quartic spectrum analysis. Firstly, three vital feature parameters of signal, which are the maximum value of the spectral power density of the normalized-centered instantaneous amplitude $R_{\max }$, the standard deviation of the absolute value of the centered nonlinear component of the instantaneous phase, evaluated over the nonweak intervals $\delta_{a p}$, and the average value of the instantaneous amplitude $E_{a}$, are extracted. Secondly, the extracted parameters are compared with the thresholds. Lastly, by analyzing the quartic spectrum peak proportion, the recognition of QPSK modulation can be realized. The simulation results show that the proposed method can recognize QPSK modulation effectively in less phase space samples and it is much more accurate than the traditional method based on feature parameters.

\section{Conflict of Interests}

The authors declare that there is no conflict of interests regarding the publication of this paper.

\section{Acknowledgments}

The research is supported by New Century Program for Excellent Talents of Ministry of Education of China (NCET11-1013), the State Key Laboratory of Rail Traffic Control and Safety (Contract no. RCS2012 K012), Beijing Jiaotong University, and Open Fund of Information Network and Information Countermeasure Technology, Key Laboratory of Liaoning Province Shenyang Ligong University.

\section{References}

[1] L. Stanković, I. Djurović, S. Stanković, M. Simeunović, S. Djukanović, and M. Daković, "Instantaneous frequency in time-frequency analysis: enhanced concepts and performance of estimation algorithms," Digital Signal Processing, vol. 35, pp. $1-13,2014$.

[2] A. I. R. Fontes, A. D. M. Martins, L. F. Q. Silveira, and J. C. Principe, "Performance evaluation of the correntropy coefficient in automatic modulation classification," Expert Systems with Applications, vol. 42, no. 1, pp. 1-8, 2015.

[3] A. K. Nandi and E. E. Azzouz, Automatic Modulation Recognition of Communication Signals, Kluwer Academic Publishers, Amsterdam, The Netherlands, 1996.

[4] L. Que and Q. Feng, "Study on phase unwrapping threshold of digital signal identification," Signal Processing, vol. 26, no. 1, pp. $56-59,2010$.

[5] H.-D. Liu, H.-X. Zhang, and P.-F. He, "Study on hybrid pattern recognition algorithm for modulated signals," The Journal of China Universities of Posts and Telecommunications, vol. 21, pp. 106-109, 2014.

[6] E. Kabalci, Y. Kabalci, and I. Develi, "Modelling and analysis of a power line communication system with QPSK modem for renewable smart grids," International Journal of Electrical Power and Energy Systems, vol. 34, no. 1, pp. 19-28, 2012.

[7] J. Huang, R. Li, Y. Wang, and C. Wu, "High-sensitivity acquisition algorithm of QPSK-modulate BeiDou B1 signal," Aeronautical Computing Technique, vol. 42, no. 5, pp. 38-42, 2012. 
[8] G. Luo, B. Yang, Z. Qiu, and Y. Li, Data Link-Connection of Information System and Weapon System, National Defense Industry, 2010.

[9] J. Chen, H. Liu, and S. Chen, "A method for channel estimation and digital modulation identification based on higher-order cumulants," Computer System Application, no. 11, pp. 172-175, 2009.

[10] S. Cao, Z. Ye, N. Hu, and X. Xu, "DOA estimation based on fourth-order cumulants in the presence of sensor gain-phase errors," Signal Processing, vol. 93, no. 9, pp. 2581-2585, 2013.

[11] N. Madhavan, A. P. Vinod, A. S. Madhukumar, and A. K. Krishna, "Spectrum sensing and modulation classification for cognitive radios using cumulants based on fractional lower order statistics," International Journal of Electronics and Communications, vol. 67, no. 6, pp. 479-490, 2013.

[12] P. W. J. Van Eetvelt, S. J. Shepherd, and S. K. Barton, "The distribution of peak factor in QPSK multi-carrier modulation," Wireless Personal Communications, vol. 2, no. 1-2, pp. 87-96, 1995.

[13] A. E. Sherme, "A novel method for automatic modulation recognition," Applied Soft Computing, vol. 12, no. 1, pp. 453-461, 2012.

[14] Y. Li, G.-T. Li, and G.-Q. Yang, "Automatic digital modulation recognition algorithm of communication signals," Journal of Electronics \& Information Technology, vol. 27, no. 2, pp. 197-201, 2005.

[15] H. Fan, Z. Yang, and Z. Cao, "Automatic recognition for common used modulations in satellite communication," Journal of China Institute of Communications, vol. 25, no. 1, pp. 140-149, 2004. 


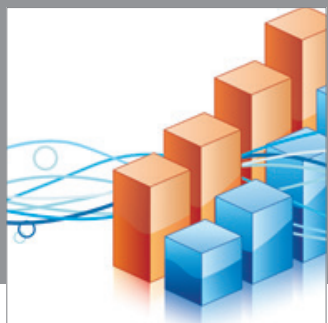

Advances in

Operations Research

mansans

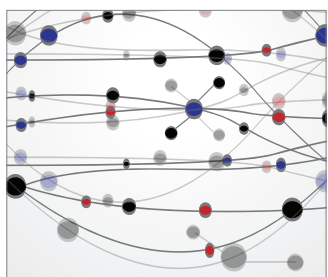

The Scientific World Journal
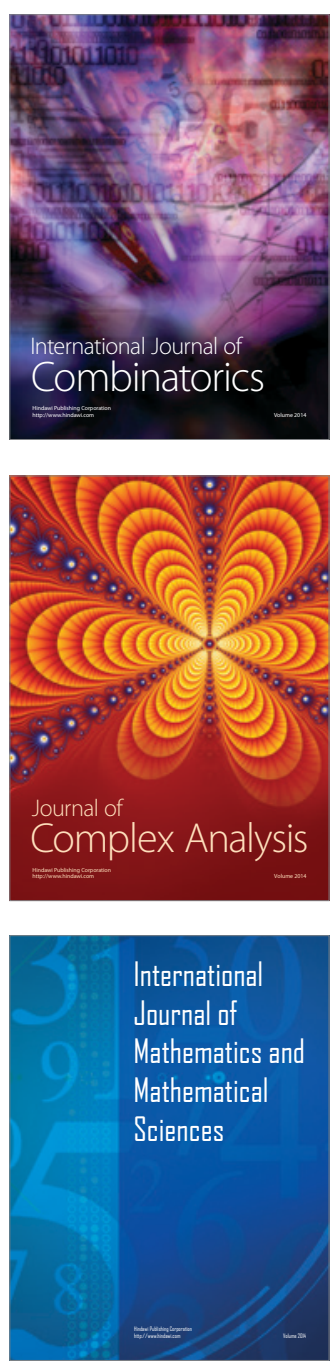
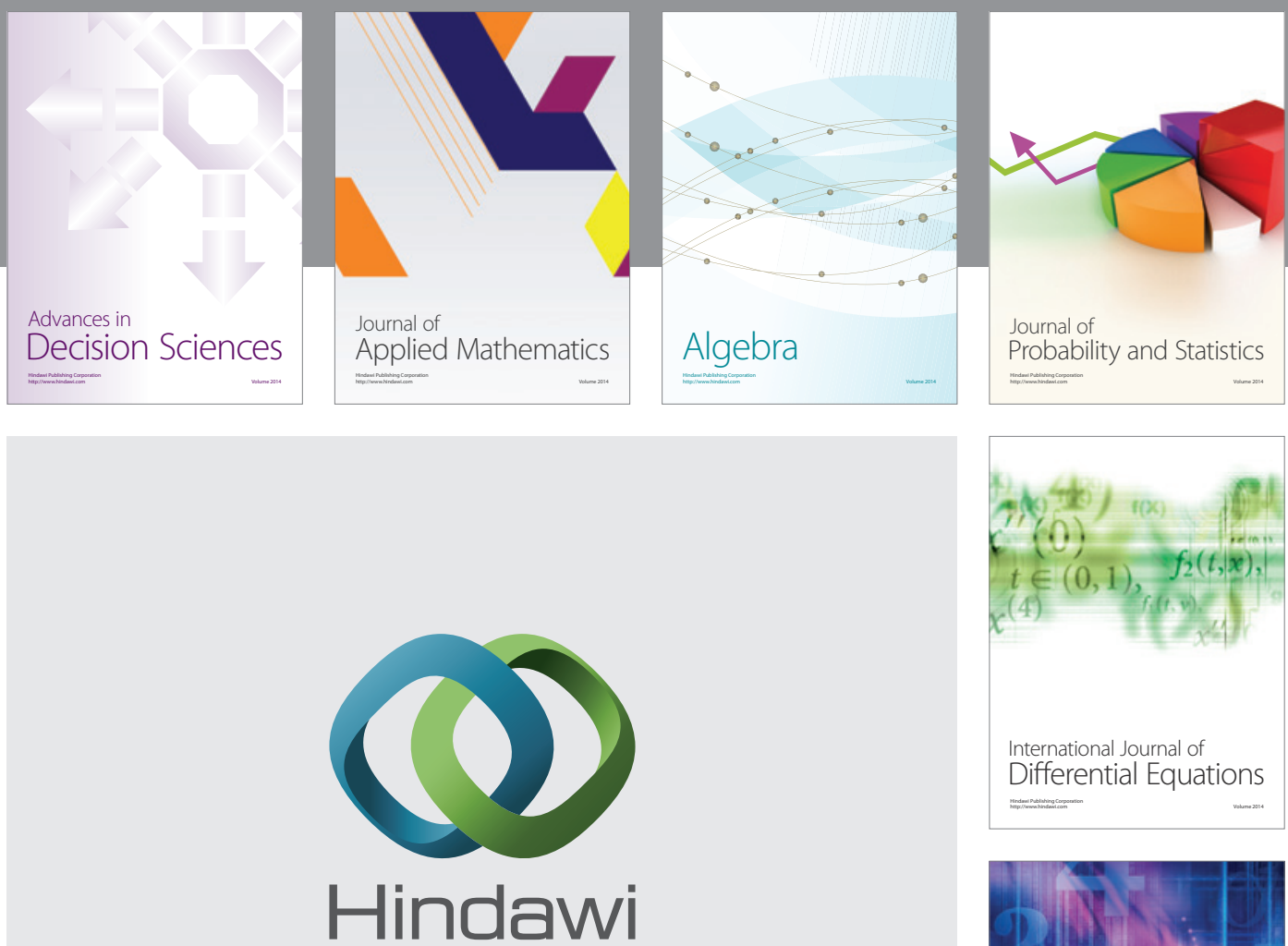

Submit your manuscripts at http://www.hindawi.com
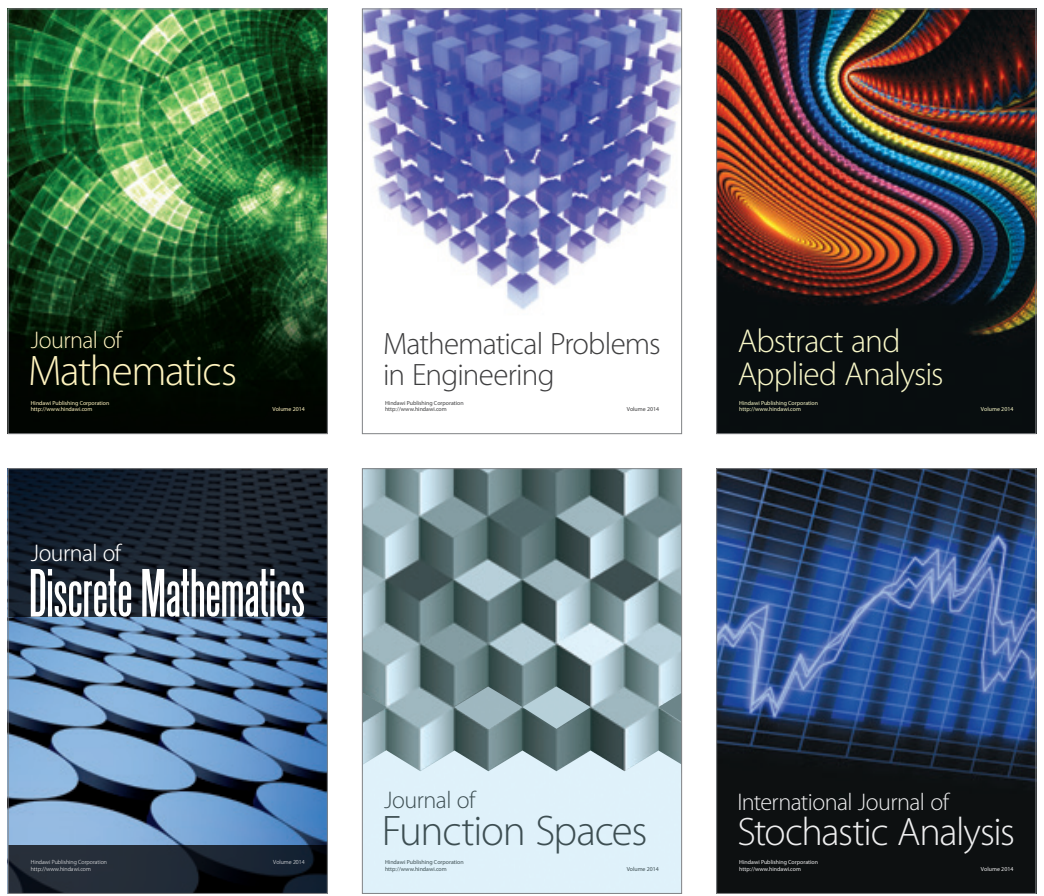

Journal of

Function Spaces

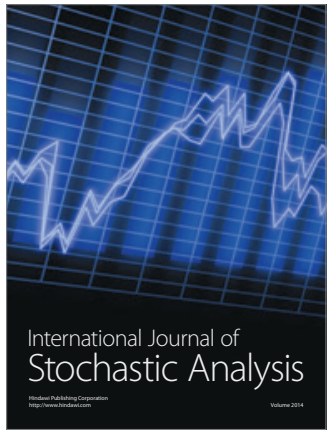

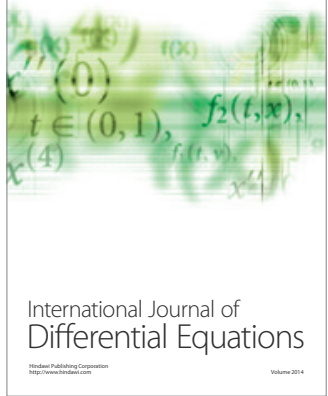
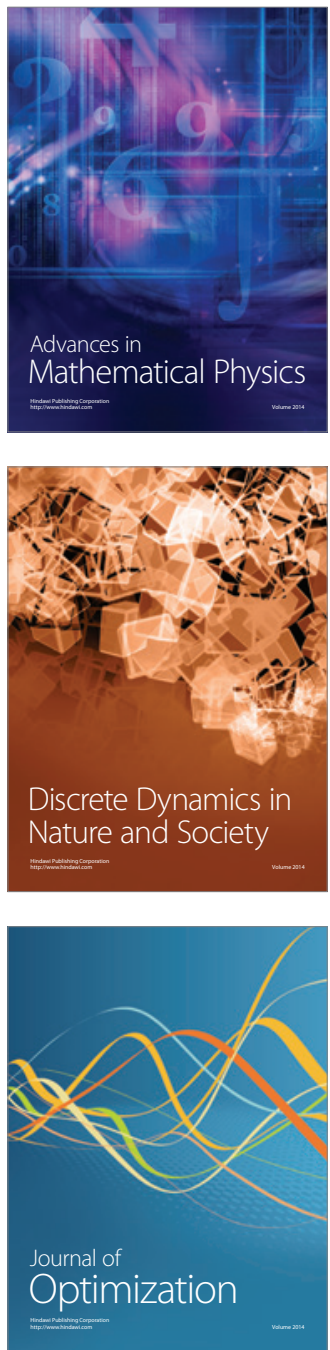